Linköping Studies in Science and Technology

Licentiate thesis No. 1912

Large deviations of

condition numbers of

random matrices

Denise Uwamariya 

Linköping Studies in Science and Technology

Licentiate Thesis No. 1912

\title{
Large deviations of condition numbers of random matrices
}

\author{
Denise Uwamariya
}

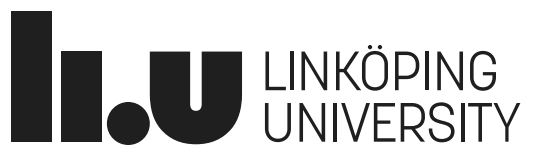

Linköping University

Department of Mathematics

Division of Mathematical Statistics

SE-581 83 Linköping, Sweden

Linköping 2021 
This is a Swedish Licentiate's Thesis

Swedish postgraduate education leads to a doctor's degree and/or a licentiate's degree. A doctor's degree comprises 240 ECTS credits (4 years of full-time studies). A licentiate's degree comprises 120 ECTS credits.

(c) EY-NC This work is licensed under a Creative Commons AttributionNonCommercial 4.0 International License.

\section{Edition 1:1}

(C) Denise Uwamariya, 2021

ISBN 978-91-7929-618-6

ISSN 0280-7971

Published articles have been reprinted with permission from the respective copyright holder.

Typeset using LATEX

Printed by LiU-Tryck, Linköping 2021 


\begin{abstract}
Random matrix theory has found many applications in various fields such as physics, statistics, number theory and so on. One important approach of studying random matrices is based on their spectral properties. In this thesis, we investigate the limiting behaviors of condition numbers of suitable random matrices in terms of large deviations.

The thesis is divided into two parts. Part I, provides to the readers an short introduction on the theory of large deviations, some spectral properties of random matrices, and a summary of the results we derived, and in Part II, two papers are appended. In the first paper, we study the limiting behaviors of the 2-norm condition number of $p \times n$ random matrix in terms of large deviations for large $n$ and $p$ being fixed or $p=p(n) \rightarrow \infty$ with $p(n)=o(n)$. The entries of the random matrix are assumed to be i.i.d. whose distribution is quite general (namely subGaussian distribution). When the entries are i.i.d. normal random variables, we even obtain an application in statistical inference. The second paper deals with the $\beta$-Laguerre (or Wishart) ensembles with a general parameter $\beta>0$. There are three special cases $\beta=1, \beta=2$ and $\beta=4$ which are called, separately, as real, complex and quaternion Wishart matrices. In the paper, large deviations of the condition number are achieved as $n \rightarrow \infty$, while $p$ is either fixed or $p=p(n) \rightarrow \infty$ with $p(n)=o(n / \ln (n))$.
\end{abstract}





\section{Acknowledgments}

As a PhD student at Linköping University (LiU), I got supports from many different people from inside and outside LiU and may this be a good opportunity to thank all of them.

First of all, I would like to express my sincere gratitude to the LiU for giving me a study opportunity in the department of mathematics (MAI) and Swedish International Development Cooperation Agency (Sida) through Rwanda-Sweden bilateral programme for providing me a financial support. Secondly, to my supervisor Xiangfeng Yang whose insight and knowledge into the subject matter steered me through this research and always be available for questions, discussions, supports and comments.

To my two co-supervisors Martin Singull and Dietrich von Rosen for working together with me and my supervisor as a team, for their advice, suggestions and corrections.

Let me extend my thanks to all lecturers at MAI, administrative staffs, Theresa Lagali, who have been helpful in too many things, especially in my first period. Additionally, I would like to express gratitude to all my colleague PhD students specially my office-mate Umunoza Gasana Emelyne for supports and discussions we had in the office especially a lot of laughs. I would like also to thank the University of Rwanda (UR), College of Science and Technology (CST) for study leave and supports.

Most importantly, I wish to thank my loving and supportive husband, Billy Second Niyinzi, and our wonderful son Derwin Seely Ganji Niyinzi who provide unending inspiration.

And finally, I am very thankful to all my family members and friends.

Thank you all. 



\section{Contents}

$\begin{array}{ll}\text { Abstract } & \text { iii }\end{array}$

Acknowledgments $\quad$ v

Contents vii

$\begin{array}{ll}\text { List of Figures } & \text { ix }\end{array}$

1 Introduction $\quad \mathbf{1}$

1.1 Condition numbers . . . . . . . . . . . . . . . . 1

1.2 Wishart ensembles with i.i.d. sub-Gaussian entries . . . . . . . 2

$1.3 \beta$-Laguerre (or Wishart) ensembles . . . . . . . . . . . . . 4

1.4 Summary of the papers . . . . . . . . . . . . . 6

2 Theoretical background 9

2.1 Rare events and large deviations . . . . . . . . . . . . . . 9

2.1.1 Large deviation principle . . . . . . . . . . . . 11

2.2 Cramér's theorem in $\mathbb{R} \ldots \ldots 12$

2.3 Gärtner-Ellis theorem . . . . . . . . . . . . . . . . . . . . 14

3 Contribution of the thesis and future work 17

3.1 Contribution of our research . . . . . . . . . . . . 17

3.2 Open problems and future work ............ 18

$\begin{array}{ll}\text { Bibliography } & 21\end{array}$

$\begin{array}{lr}\text { Paper I } & 27\end{array}$

$\begin{array}{ll}\text { Paper II } & 49\end{array}$ 



\section{List of Figures}

2.1 The behavior of pdf of sample mean $\bar{X}_{n}$ for $\mu=0, \sigma=1$ and different values of $n$ and $\alpha$. (Normal random variable case) . . . 14

2.2 The behavior of pdf of sample mean $\bar{X}_{n}$ for different values of $n$ and $\alpha$. (Bernoulli random variable case) $\ldots \ldots \ldots \ldots$ 



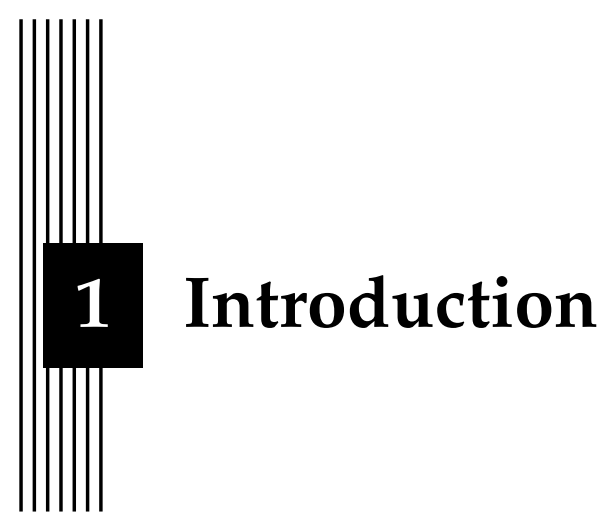

To understand the behavior of the condition number of a random matrix is important because the condition numbers play a part in numerical linear algebra and in the theory of probability in Banach spaces ([31] and [27]). Another reason is that the condition numbers relate to the so called first antieigenvalues that have applications in statistics and in many other areas [16]. For instance, one can mention one application in statistics that uses condition numbers: testing the null hypothesis that the covariance is a scalar multiple of identity. The union-intersection test method in [29, Section 7.4] suggests that we reject the null hypothesis for large values of the condition number. The question of interest here is how to calculate the $p$-value? The answer to the question, is to investigate the null distribution of the sample condition number. This thesis is concerned with the asymptotic properties of condition numbers of suitable random matrices.

\subsection{Condition numbers}

Let $\mathbf{X}$ be $p \times n$ random matrix (here, $p, n \geqslant 2$ and are any two positive integers) and let random variables $X_{i j}, 1 \leqslant i \leqslant p, 1 \leqslant j \leqslant n$, be the entries of $\mathbf{X}$. 
The 2-norm condition number $\kappa(p, n)$ of $\mathbf{X}$ is defined as

$$
\kappa(p, n)=\sigma_{\max } / \sigma_{\min }
$$

where $\sigma_{\max }$ and $\sigma_{\min }$ are the maximal and minimal singular values of $\mathbf{X}$. If we look at the eigenvalues of $p \times p$ square random matrix $\mathbf{X X}^{T}$, then $\sigma_{\max }(\mathbf{X})=\left(\lambda_{\max }\left(\mathbf{X X}^{T}\right)\right)^{1 / 2}$ and $\sigma_{\min }(\mathbf{X})=\left(\lambda_{\min }\left(\mathbf{X X}^{T}\right)\right)^{1 / 2}$. Therefore, with the relation the condition number has an equivalent form $\kappa(p, n)=$ $\sigma_{\max } / \sigma_{\min }=\left(\lambda_{\max } / \lambda_{\min }\right)^{1 / 2}$ with $\lambda_{\max }$ and $\lambda_{\min }$ denoting the maximal and minimal eigenvalues of the square random matrix $\mathbf{X} \mathbf{X}^{T}$.

To study the asymptotic properties of condition numbers of random matrices there are number of points to consider. The first point is the matrix size. In the literature, we found studies of condition numbers of rectangular random matrices (this is the case when $p<n$ ) and of square random matrices (when $p=n$ ); (see for instance $[1,5,12,22]$ for rectangular random matrices and $[11,27,30]$ for square random matrices). Another point is on the distributions of entries, for example random matrices whose entries are standard normal random variables (see results in [1, 5, 11, 12]) and entries being other (in particular those with discrete as entries) random variables $([22,27,30])$.

The aim of this thesis is to study limiting behaviors of $P(k(p, n) \geqslant c)$ with $c \geqslant 1$, in the framework of $k(p, n)$ approaching 1 for large $n$ (and possibly for large $p$ at the same time as well) of suitable random matrices $\mathbf{X}$ of size $p \times n$ whose entries satisfy some conditions. Since the condition numbers are invariant under matrix transpose, throughout this thesis we assume $p \leqslant n$ without loss of generality.

Note that in general, it is often straightforward to study random matrices whose entries are standard normal random variables since there is an expression for the joint density function of the eigenvalues which cannot be found for discrete random matrices. When the entries of random matrices are sub-Gaussian random variables, it is possible to control its moments, tail estimates and moment generating function explicitly which can be used in the derivation results on condition number.

Now we define two classes of random matrices for which we will study the corresponding limiting behaviors of the condition numbers.

\subsection{Wishart ensembles with i.i.d. sub-Gaussian entries}

Definition 1.1 (Sub-Gaussian random variable). A random variable $X$ with parameters $K_{i}, 1 \leqslant i \leqslant 3$ differing from each other by at most an absolute 
constant factor ([35, Lemma 5.5]), is sub-Gaussian if it satisfies one of the following three equivalent properties:

(i) Tails: $P(|X|>t) \leqslant \exp \left\{1-t^{2} / K_{1}^{2}\right\}$ for all $t \geqslant 0$;

(ii) Moments: $\left(E|X|^{p}\right)^{1 / p} \leqslant K_{2} \sqrt{p}$ for all $p \geqslant 1$;

(iii) Super-exponential moment: $E \exp \left\{X^{2} / K_{3}^{2}\right\} \leqslant e$;

where $E$ denotes the expectation. If $E(X)=0$, then (i)-(iii) are also equivalent to the following:

(iv) Moment generating function: $E \exp \{t X\} \leqslant \exp \left\{t^{2} K_{4}^{2}\right\}$ for all $t \in \mathbb{R}$ for some constant $K_{4}$.

Consider a $p \times n$ random matrix $\mathbf{X}$ whose entries $X_{i j}, 1 \leqslant i \leqslant p, 1 \leqslant$ $j \leqslant n$, are independent and identically distributed (or i.i.d. in short) subGaussian random variables. The class of sub-Gaussian random variables is vast containing normal (also known as Gaussian), Bernoulli and bounded random variables. If the entries $X_{i j}, 1 \leqslant i \leqslant p, 1 \leqslant j \leqslant n$, are i.i.d. standard normal $N(0,1)$ then, $p \times p$ square matrix $\mathbf{X} \mathbf{X}^{T}$, follows a standard Wishart distribution (i.e., $\mathbf{X} \mathbf{X}^{T} \sim W_{p}(n, \mathbf{I})$ with $\mathbf{I}=\mathbf{I}_{p \times p}$ being an identity matrix), and is called the Wishart matrix.

Based on Dyson idea in 1962, the threefold way [10], square matrix $\mathbf{X X}^{T}$ can be classified into three special matrix models by considering random matrix $\mathbf{X}$ over i.i.d. entries being real, complex or quaternion normal distribution. In this setting, one can define the so called Wishart ensembles composed by three ensembles, i.e., real, complex and quaternion Wishart ensemble.

The real Wishart ensemble is the ensemble of $p \times p$ symmetric matrices $\mathbf{X} \mathbf{X}^{T}$ where $\mathbf{X}$ is real normal distribution of size $p \times n$ (i.e., entries are i.i.d. real standard normal $N(0,1))$. The complex Wishart ensemble is the ensemble of $p \times p$ hermitian matrices $\mathbf{X} \mathbf{X}^{T}$, where $\mathbf{X}$ is complex normal distribution of size $p \times n$ (i.e., entries are i.i.d. complex standard normal $\left.N^{2}(0,1)\right)$. The quaternion Wishart ensemble is the ensemble of $p \times p$ self-dual matrices $\mathbf{X} \mathbf{X}^{T}$ where $\mathbf{X}$ is quaternion normal distribution of size $p \times n$ (i.e., entries are i.i.d. quaternion standard normal $\left.N^{4}(0,1)\right)$. We have used superscript 2 to denote that for matrix $\mathbf{X}$ of complex normal distribution we have 2 real i.i.d. random variables with distribution $N(0,1)$ in the form of complex entries and 4 real i.i.d. random variables with distribution $N(0,1)$ in the form of quaternion complex entries for matrix $\mathbf{X}$ of quaternion normal distribution.

These ensembles are known as Wishart ensembles in the literature and the name comes from statistician John Wishart, who first proposed a matrix 
model named the real Wishart model in 1928 [36]. The Wishart ensembles are labeled by the indexes $\beta=1,2,4$ respectively, to indicate random matrces $\mathbf{X}$ over i.i.d. entries being real, complex or quaternion normal distribution.

The spectral properties of the Wishart matrices have been studied widely and it is well known in the literature that, the joint probability density function of eigenvalues $\lambda=\left(\lambda_{\max }=\lambda_{1}, \ldots, \lambda_{p}=\lambda_{\min }\right)$ of $\mathbf{X} \mathbf{X}^{T}$ is given by

$$
\begin{aligned}
& f(\lambda ; p, n, \beta)= \\
& c(p, n, \beta) \cdot \exp \left\{-\sum_{i=1}^{p} \lambda_{i} / 2\right\} \cdot \prod_{1 \leqslant i<j \leqslant p}\left|\lambda_{i}-\lambda_{j}\right|^{\beta} \cdot \prod_{i=1}^{p} \lambda_{i}^{\beta(n-p+1) / 2-1},
\end{aligned}
$$

where

$c(p, n, \beta)=2^{-\beta n p / 2} \prod_{j=1}^{p} \Gamma(1+\beta / 2)[\Gamma(1+\beta j / 2) \Gamma(\beta(n-p+j) / 2)]^{-1}, \Gamma(\cdot)$ denotes gamma function, and $\beta$ is equal to 1,2 and 4 for the real, complex, and quaternion Wishart matrix respectively (for reference see, [18] for $\beta=1$, [23] for $\beta=2$ and [12] for $\beta=4$ ).

Regarding the condition numbers, in [1] the derivation of density function of the condition numbers $\kappa(p, n)$ for Wishart random matrices was given for all $2 \leqslant p \leqslant n$ with involved forms. For large $c$, lower and upper bounds of $P(k(p, n) \geqslant c)$ for Wishart matrices can be found in [5] and [12] with fixed $p$ and $n$. In [13], the authors provided individual and jointly large deviation asymptotics for $\lambda_{\max }$ and $\lambda_{\min }$ with somewhat more general entries as $n \rightarrow \infty$. But the limiting behaviors of $P(k(p, n) \geqslant c)$ cannot be obtained from the existing results therein.

For a long time ago, researchers have shown interest in the study of Wishart ensembles for the cases $\beta=1,2$ or 4 since they correspond to the matrix models defined above. It turns out that the Wishart ensembles can be generalized to general $\beta>0$ and we recall the results below.

\section{$1.3 \beta$-Laguerre (or Wishart) ensembles}

Let $\mathbf{X}$ be a real (respectively complex or quaternion) normal random matrix of size $p \times n$ so that $\mathbf{X X}^{T}$ is a Wishart real (respectively complex or quaternion) matrix and

$$
\mathbf{x}_{\beta} \sim\left[\begin{array}{cccc}
\chi_{\beta n} & & & \\
\chi_{\beta(p-1)} & \chi_{\beta(n-1)} & & \\
& \ddots & \ddots & \\
& & \chi_{\beta} & \chi_{\beta(n-p+1)}
\end{array}\right]
$$


be bidiagonal form of $\mathbf{X}$ with independent entries for $\beta>0$, where $\chi$ denotes the chi-distribution known as the square root of $\chi^{2}$ distribution.

The work done by Dumitriu and Edelman in [9] provides real tridiagonal random matrix models $\mathbf{X}_{\beta} \boldsymbol{X}_{\beta}^{T}$ for general $\beta>0$ such that the joint probability density of eigenvalues of $\mathbf{X}_{\beta} \boldsymbol{X}_{\beta}^{T}$ has exactly the same density function $f(\lambda ; p, n, \beta)$ defined in (1.1) for $\beta>0$. Since then, researchers started to study the spectral properties of the eigenvalues of $\boldsymbol{X}_{\beta} \boldsymbol{X}_{\beta}^{T}$ since it corresponds to tridiagonal matrix models for any $\beta>0$. The studies can be classified into two categories: the study involved all eigenvalues (the global or bulk category), and the study involved the first few (i.e., the maximal and minimal) eigenvalues (the local or extremes category). The matrix models $\mathbf{X}_{\beta} \boldsymbol{X}_{\beta}^{T}$ for any $\beta>0$ are known as $\beta$-Laguerre ensembles or $\beta$-Wishart ensembles in the literature.

Let us mention some work done about the spectral properties of the eigenvalues of $\beta$-Laguerre ensembles. Results from the literature are summarized here for both categories are related to the weak convergence, laws of large numbers and large deviations. For the global (or bulk) category, in the case that $n \rightarrow \infty$ and fixed $p$ or $p=p(n) \rightarrow \infty$ with $p(n)=o\left(n^{1 / 3}\right)$, it was shown that the joint distribution of all eigenvalues of $\beta$-Laguerre ensembles converges to the joint distribution correspond to the one called $\beta$-Hermite ensembles [19, Theorem 1]. In [8, Section 6.5], for $\beta>0$, it was proven that when $p(n) / n \rightarrow \kappa \in(0,1]$ the empirical distribution of the eigenvalues almost surely converges to the Marčenko-Pastur distribution but when $p(n) / n \rightarrow 0$ empirical distribution in probability converges to the semi-circle distribution [19, Proposition 3]. The result about the large deviation principle for the empirical distribution of eigenvalues of $\beta$-Laguerre ensembles was also established in the literature. For $\beta=1$, with $p(n) / n \rightarrow \kappa \in(0,1]$ large deviation principle for the empirical distribution was derived in [24], and in [19, Theorem 4] for $\beta>0$ with $p(n)=o\left(n^{1 / 2}\right)$.

Similarly to the local (or the extremes) category, the result such as the weak convergence of the maximal and minimal eigenvalues of $\beta$-Laguerre ensembles was obtained and it was shown that for general $\beta>0$ the maximal and minimal eigenvalues converge to general $\beta$ Tracy-Widom law. The weak convergence of the maximal eigenvalue can be found in [20], for $\beta=1$ and for $\beta=2$ in [21], and in [4] for minimal eigenvalue for $\beta=2$. For $\beta>0$, the expression of weak convergence result of the maximal eigenvalue was stated in [26] from which for $\beta>0$, the weak convergence result of the minimal eigenvalue follows. The study about the laws of large numbers of the 
extreme maximal and minimal eigenvalues can be obtained from the results in [19, Theorem 2 and 3] for fixed $p$, and when $p=p(n) \rightarrow \infty$ as $n \rightarrow \infty$ under the assumption $p(n) / n \rightarrow \kappa \in[0,1]$ or $\kappa \in[0,1)$ the results can be obtained in [26]. By imposing some restrictions on $p$ and $n$, for general $\beta>0$ large deviations for the maximal and minimal eigenvalues of $\beta$-Laguerre ensembles has been given in [19].

In the literature there is no study about the ratio of the maximal and minimal eigenvalues of $\beta$-Laguerre ensembles (namely, the condition numbers $\kappa(p, n)$ of $\left.\mathbf{X}_{\beta}\right)$, therefore in this thesis we study the limiting behaviors of $\kappa(p, n)$ in terms of large deviations.

\subsection{Summary of the papers}

The thesis is a summary of research work done, two papers appended provide new results in the literature and here we summarized the results from each paper.

Paper I: Large deviation asymptotics of condition numbers of random matrices

The paper deals with the case when the entries of random matrix $\mathbf{X}$ are assumed to be sub-Gaussian random variables with zero mean and unit variance, and studies the limiting behaviors of the condition number $k(p, n)$ in terms of large deviations for large $n$, with $p$ being fixed or $p=p(n) \rightarrow \infty$ with $p(n)=o(n)$. The results of the paper are summarized in Theorem 1.2 and Corollary 1.3.

Theorem 1.2. Suppose that the entries $X_{i j}, 1 \leqslant i \leqslant p, 1 \leqslant j \leqslant n$ of $\mathbf{X}$ are i.i.d. subGaussian with zero mean and unit variance. Then for any $c \geqslant 1$ it holds that for fixed $p$,

$$
\lim _{n \rightarrow \infty} n^{-1} \ln P\left(k^{2}(p, n) \geqslant c\right)=-I_{p, 0}(c),
$$

and for $p=p(n) \rightarrow \infty$ with $p(n)=o(n)$,

$$
\lim _{n \rightarrow \infty} n^{-1} \ln P\left(k^{2}(p, n) \geqslant c\right)=-I_{\infty, 0}(c),
$$

where $I_{p, 0}$ and $I_{\infty, 0}$ are properly defined rate functions.

Since standard normal distribution is sub-Gaussian, a very special case of Theorem 1.2 is the real central Wishart matrix $W_{p}\left(n, n^{-1} \mathbf{I}\right)$ for which the entries $X_{i j}, 1 \leqslant i \leqslant p, 1 \leqslant j \leqslant n$ of $\mathbf{X}$ are i.i.d. standard normal $N(0,1)$. 
Corollary 1.3. Suppose that the entries $X_{i j}, 1 \leqslant i \leqslant p, 1 \leqslant j \leqslant n$ of $\mathbf{X}$ are i.i.d. standard normal $N(0,1)$. Then for any $c \geqslant 1$ it holds that

$$
\lim _{n \rightarrow \infty} n^{-1} \ln P\left(k^{2}(p, n) \geqslant c\right)=-2^{-1} \ln \left[(c+1)^{2} /(4 c)\right]
$$

when $p$ is fixed or $p=p(n) \rightarrow \infty$ with $p(n)=o(n)$.

With the proof of Corollary 1.3 we were able to provide one application in statistics: To test the null hypothesis that the covariance is a scalar multiple of identity using union-intersection test method.

Paper II: On the ratio of extremal eigenvalues of $\beta$-Laguerre ensembles The paper considers $\beta$-Laguerre ensembles for any $\beta>0$, and studies the limiting behaviors of the ratio of the extremal eigenvalues (namely, the condition number). The following result was obtained.

Theorem 1.4. For $\beta$-Laguerre ensembles whose joint probability density of eigenvalues is given by (1.1), suppose that $p$ is either fixed or $p=p(n) \rightarrow \infty$ as $n \rightarrow \infty$ with $p=o(n / \ln n)$. Then it holds that, for any $\beta>0$ and $c \geqslant 1$,

$$
\lim _{n \rightarrow \infty} n^{-1} \ln P\left(k^{2}(p, n) \geqslant c\right)=-(\beta / 2) \ln \left[(c+1)^{2} /(4 c)\right] .
$$





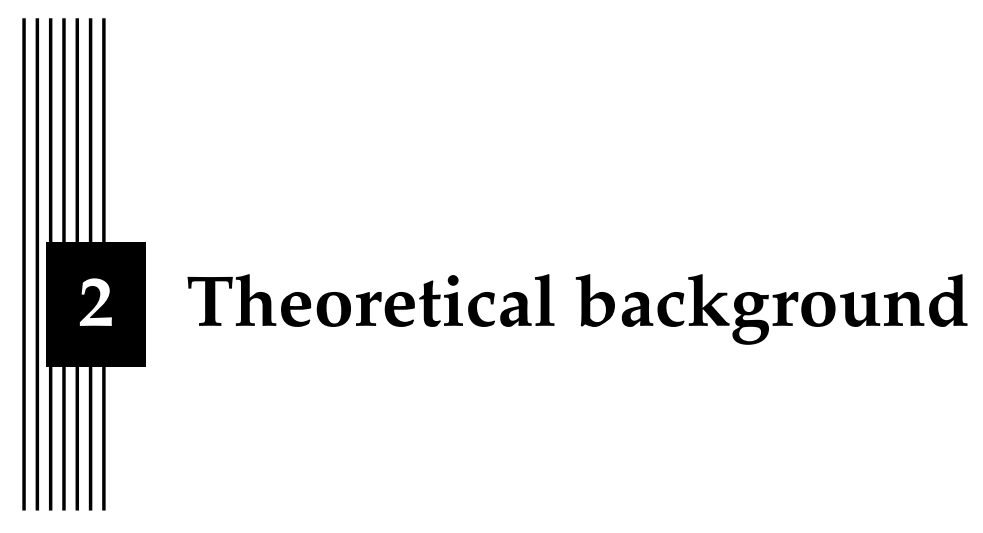

The theory of large deviations studies probabilities of rare events, and it started by the work of Boltzmann a physicist in 1870 and the work of Cramér in 1938 [6]. Later on, the general abstract structure of large deviations was given by the work of Varadhan in 1966 [33], and Wentzell and Freidlin in 1970 [15].

For suitable $p \times n$ random matrices, this thesis aims to study the limiting behaviors of the condition numbers $\kappa(p, n)$ for $n \rightarrow \infty$ and possibly $p=$ $p(n) \rightarrow \infty$ as well, in terms of large deviations.

This chapter provides an overview of the theory of large deviations. It includes, some definitions and mathematical concepts to understand the basic tools to handle the probabilities of rare events using large deviations and it can be used as a joint introduction to appended papers as part of the thesis. More details about the theory of large deviations can be found in $[7,17,34]$.

\subsection{Rare events and large deviations}

In summary one can define rare or unlikely events shortly as events that occur with small frequency. Mathematically, the events $A_{\alpha}$ (depending on suitable parameter(s) $\alpha$ ) are rare if $P\left(A_{\alpha}\right) \rightarrow 0$ as $\alpha \rightarrow \alpha_{0}$. 
In real world we find various examples of rare events underlying different phenomena, such as asteroid impacts, major earthquakes, tsunamis, a statistical estimator gives a wrong information, floods, bankruptcies, acts of terrorism, epidemic or pandemic disease spread, global warming-related changes in climate and weather, etc and those rare events can cause huge impact to the society. The study of rare events is based on the study of how fast the probabilities of rare events converge to zero. One way to address this convergence is to use the theory of large deviations.

The theory of large deviations is a theory that studies and deals with the techniques for estimating probabilities of rare or unlikely events. The first question might be why interested in unusual or unlikely events but the point is that rare events can have a huge negative impact as real world examples show. It is not so realistic to imagine or to expect that something will happen and avoid all rare events but it may be possible to minimize effect of rare events, and also possibly make rare events rarer or infrequent.

To clarify the idea behind the theory of large deviations, let us consider a specific example. Suppose that we have i.i.d. random variables $X_{1}, \ldots X_{n}$, where $E\left(X_{1}\right)=\mu \in \mathbb{R}$ and $V\left(X_{1}\right)=\sigma^{2}=E\left[\left(X_{1}-\mu\right)^{2}\right] \in(0, \infty)$, and let

$$
\bar{X}_{n}=n^{-1} \sum_{i=1}^{n} X_{i}
$$

be their sample mean. In this respect, we can mention two laws that play key roles in probability and statistics: (1) Law of Large Numbers (LLN) states that $\bar{X}_{n} \rightarrow \mu$, in probability as $n \rightarrow \infty$, and (2) Central Limit Theorem (CLT) states that $\sqrt{n}\left(\bar{X}_{n}-\mu\right) \Longrightarrow \sigma Z$, in distribution as $n \rightarrow \infty$, here $Z$ is a standard normal random variable.

The theory of large deviations complements the LLN and CLT since these two laws say little or nothing about rate of convergence. For instance, if $\alpha>0$ by LLN

$$
P\left(\left|\bar{X}_{n}-\mu\right| \geqslant \alpha\right) \rightarrow 0 \text { as } n \rightarrow \infty,
$$

and the theory of large deviations extends the LLN to provide information about how fast this probability converges to zero as $n \rightarrow \infty$.

According to CLT we have: for fixed $\alpha$,

$$
\lim _{n \rightarrow \infty} P\left(\sqrt{n}\left(\bar{X}_{n}-\mu\right) \geqslant \alpha\right)=\left(2 \pi \sigma^{2}\right)^{-1 / 2} \int_{\alpha}^{\infty} \mathrm{e}^{-(x-\mu)^{2} / 2 \sigma^{2}} \mathrm{~d} x .
$$

However, CLT is not so informative when $\alpha=\alpha(n)$ depends on $n$ as well. For example, if $\alpha(n)=o(\sqrt{n})$ we have moderate deviation and under good 
condition the above limit still holds. But if $\alpha(n)=O(\sqrt{n})$ then it is a large deviation result.

The study of rare events $\left\{\left|\bar{X}_{n}-\mu\right| \geqslant \alpha\right\}$ is a typical example in the insurance company.

Example 2.1. Suppose that the insurance company would like to decide monthly premium $\alpha$, let $X_{i}$ denote the claim amount for each month say $i=1, \ldots, n$, and $\sum_{i=1}^{n} X_{i}$ be total claim amount. For the insurance company to succeed, it is assumed that the total amount of premium earned exceeds the total amount of claim payed out. To achieve that the company should choose a good premium $\alpha$ so that the $P\left(\sum_{i=1}^{n} X_{i} \geqslant n \alpha\right)$ is very small and it is expected that the probability decays exponentially in $n$.

Back to random matrices $\mathbf{X}$ with i.i.d. entries, laws of large numbers of the extreme eigenvalues $\lambda_{\max }$ and $\lambda_{\min }$ have been obtained in the following forms:

$$
\lambda_{\max } \rightarrow\left(1+\kappa^{1 / 2}\right)^{2} \quad \text { and } \quad \lambda_{\min } \rightarrow\left(1-\kappa^{1 / 2}\right)^{2}
$$

in probability under the assumption $p / n \rightarrow \kappa \in[0,1]$ as $n \rightarrow \infty$ (see for instance [11, Lemma 4.1] for Wishart matrices and [2,3] for general matrices) and similar results holds for $\beta$-Laguerre ensembles $([19,26])$. Therefore when $p$ is either fixed or $p(n)=o(n)$ this is the case $\kappa=0$, it always holds that $k(p, n)$ converge to 1 in probability. Then, the probability of the condition number $\{k(p, n) \geqslant c\}$ with $c \geqslant 1$ converges to 0 as $n \rightarrow \infty$. We shall study this rate of convergence using the theory of large deviations.

To express the limiting behaviors of the condition numbers $\kappa(p, n)$ in terms of large deviations, Large Deviation Principle (LDP) is recalled bellow.

\subsubsection{Large deviation principle}

The large deviation principle provides the limiting behavior of a sequence of probability measures in terms of rate function through lower and upper bound ([7, Section 1.2], [14, Chap 2] and [17, Lemma I.14]).

Let $\left\{P_{n}\right\}$ be a sequence of probability measures on $(\mathbb{R}, \mathcal{B}(\mathbb{R}))$. It is said that the sequence of probability measures $\left\{P_{n}\right\}$ satisfies a large deviation principle with a good rate function $I: \mathbb{R} \rightarrow[0, \infty]$ and speed $n \in \mathbb{R}, n \rightarrow \infty$ if

(i) $0 \leqslant I(\alpha) \leqslant \infty, \forall \alpha \in \mathbb{R}$,

(ii) I is lower semi-continuous, 
(iii) For any $x<\infty$, the set $\{\alpha \in \mathbb{R}: I(\alpha) \leqslant x\}$ is compact in $(\mathbb{R}, \mathcal{B})$,

(iv) For any closed set $F \subset \mathbb{R}$,

$$
\limsup _{n \rightarrow \infty} n^{-1} \ln P_{n}(F) \leqslant-\inf _{\alpha \in F} I(\alpha)
$$

(v) For any open set $G \subset \mathbb{R}$,

$$
\liminf _{n \rightarrow \infty} n^{-1} \ln P_{n}(G) \geqslant-\inf _{\alpha \in G} I(\alpha) .
$$

In 1938 Harald Cramér [6] was interested in the kind of insurance problems define in Example 2.1 and he studied the question of how fast the probability $P\left(\sum_{i=1}^{n} X_{i} \geqslant n \alpha\right)$ tends to zero where $X_{1}, \ldots X_{n}$ are i.i.d. random variables and then he arrived to the result known as Cramér's theorem in the literature. The result carried out by Cramér is considered as the earliest systematic treatment of larger deviations and can be found in many text books for instance see ([7, Section 2], [17, Chap I] and [34, Section 3]).

To study asymptotic lower and upper bound of $P(k(p, n) \geqslant c)$ with $c \geqslant 1$, for $n \rightarrow \infty$, we associate the probability of the condition number $\{k(p, n) \geqslant$ $c$ \} with one containing $n$ i.i.d. random variables and then, apply Cramér's theorem. In Section 2.2 we define the Cramér's theorem for i.i.d. case. For possibly non-i.i.d. case, Cramér's theorem cannot be applied directly and in Section 2.3 we state Gärtner-Ellis theorem for this case.

\subsection{Cramér's theorem in $\mathbb{R}$}

Cramér's theorem estimates the large deviations associated with i.i.d. random variables. Let us consider again the sequence of i.i.d. random variables $\left\{X_{i}\right\}_{i \geqslant 1} \in \mathbb{R}$ and consider their sample mean $\bar{X}_{n}$ for all $n \geqslant 1$ with $E\left[X_{1}\right]=\mu \in \mathbb{R}, V\left(X_{1}\right)=\sigma^{2} \in(0, \infty)$, and

$$
M(\theta)=E \exp \left\{\theta X_{1}\right\}<\infty, \quad \forall \theta \in \mathbb{R} .
$$

Here the function $M(\cdot)$ in $(2.1)$ is called the moment generating function and its logarithm $(\Lambda(\theta)=\ln M(\theta))$ is called the logarithmic moment generating function or the cumulant generating function.

The Fenchel-Legendre transform of $M(\theta)$ is given as

$$
I(\alpha)=\sup _{\theta \in \mathbb{R}}[\theta \cdot \alpha-\Lambda(\theta)], \quad \forall \alpha \in \mathbb{R} .
$$


The Cramér's theorem [25, Theorem 2.3], says that if $\left\{X_{i}\right\}$ are real valued i.i.d. having finite moment generating function $M(\theta)$ define in (2.1). Then, for all $\alpha>\mu$,

$$
\lim _{n \rightarrow \infty} n^{-1} \ln P\left(\bar{X}_{n} \geqslant \alpha\right)=-I(\alpha),
$$

where $I(\alpha)$ is the rate function defined in (2.2).

To understand what Cramér's theorem says and to calculate the probabilities of rare events using Cramér's theorem, let us consider two examples. Example 2.2. Let assume that $\left\{X_{i}\right\}_{i \geqslant 1}$ are i.i.d. $N\left(\mu, \sigma^{2}\right)$ random variables. Then,

$$
M(\theta)=E \exp \left\{\theta X_{1}\right\}=\exp \left\{\mu \theta+\left(\sigma^{2} / 2\right) \theta^{2}\right\},
$$

which implies that

$$
I(\alpha)=\sup _{\theta \in \mathbb{R}}[\theta \cdot \alpha-\Lambda(\theta)]=\frac{1}{2}\left(\frac{\alpha-\mu}{\sigma}\right)^{2} .
$$

Hence, by (2.3), and for $\alpha>\mu$,

$$
\lim _{n \rightarrow \infty} n^{-1} \ln P\left(\bar{X}_{n} \geqslant \alpha\right)=-\frac{1}{2}\left(\frac{\alpha-\mu}{\sigma}\right)^{2} .
$$

If $\left\{X_{i}\right\}_{i \geqslant 1}$ are i.i.d. standard normal, the rate function is $\left(\alpha^{2} / 2\right)$.

Example 2.3. Suppose that $\left\{X_{i}\right\}_{i \geqslant 1}$ are i.i.d. Bernoulli random variables with $P\left(X_{i}=1\right)=P\left(X_{i}=-1\right)=1 / 2$.

Then,

$$
M(\theta)=E \exp \left\{\theta X_{1}\right\}=(\exp \{\theta\}+\exp \{-\theta\}) / 2,
$$

and it provides

$$
I(\alpha)= \begin{cases}1 / 2(1+\alpha) \ln (1+\alpha)+1 / 2(1-\alpha) \ln (1-\alpha) & \text { if } \alpha \in[-1,+1], \\ \infty & \text { if } \alpha \notin[-1,+1] .\end{cases}
$$

Hence, $\lim _{n \rightarrow \infty} n^{-1} \ln P\left(\bar{X}_{n} \geqslant \alpha\right)=-I(\alpha)$.

To illustrate the behavior of probability distribution of sample mean in the form of $P\left(\bar{X}_{n} \geqslant \alpha\right)$, for i.i.d. random variables being normal or Bernoulli distributed, and the corresponding rate function $I(\alpha)$ the results, we obtain in Examples 2.2 and 2.3 have been plotted for different values of $n$ and $\alpha$. See Figure 2.1 and Figure 2.2 for normal and Bernoulli random variable case respectively. The figures show that $I(\alpha)=0$ in both cases only when $\alpha$ is the most probable point (which is the limit in probability of $\bar{X}_{n}$ ). For instance, for normal case, the most probable point of $\bar{X}_{n}$ is $\mu$. 

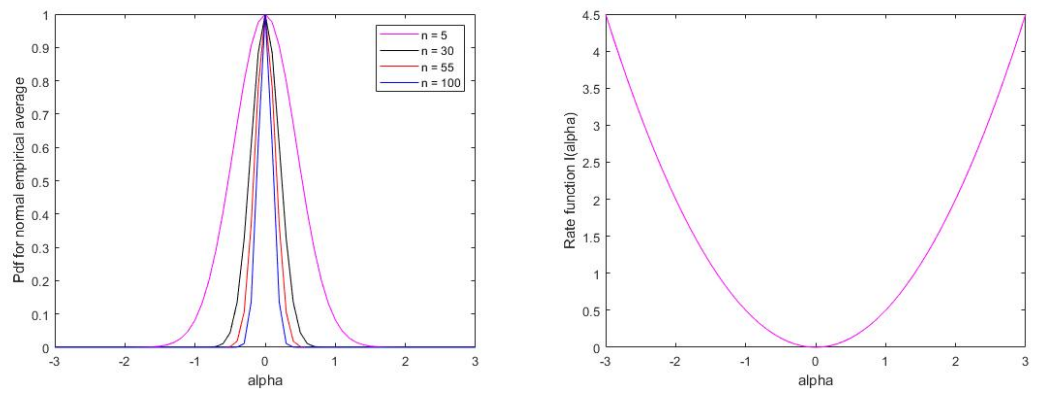

Figure 2.1: The behavior of pdf of sample mean $\bar{X}_{n}$ for $\mu=0, \sigma=1$ and different values of $n$ and $\alpha$. (Normal random variable case)
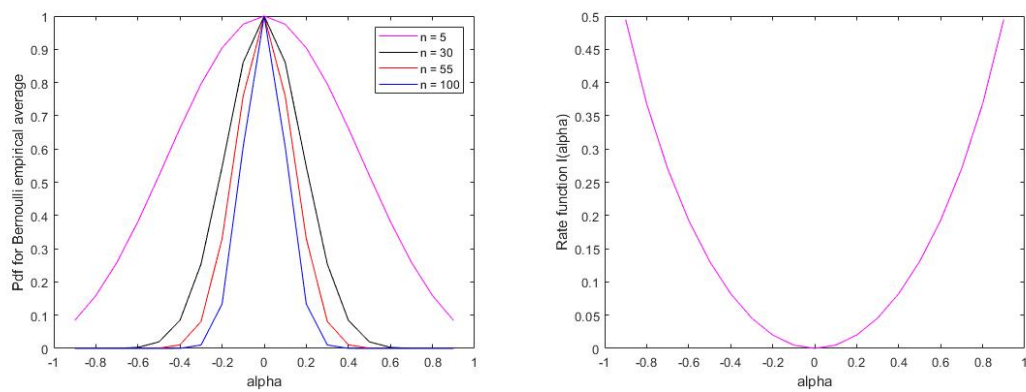

Figure 2.2: The behavior of pdf of sample mean $\bar{X}_{n}$ for different values of $n$ and $\alpha$. (Bernoulli random variable case)

\subsection{Gärtner-Ellis theorem}

Cramér's theorem works well for the i.i.d. case. For non-i.i.d. case, we shall apply the Gärtner-Ellis theorem [7, Section 2.3]. Let $Z_{n} \in \mathbb{R}$ be a sequence of random variables and the logarithmic moment generating function is given as

$$
\Lambda_{n}(\theta)=\ln E \exp \left\{\theta Z_{n}\right\}
$$

Assume that for each $\theta \in \mathbb{R}$ the limit $\Lambda(\theta)=\lim _{n \rightarrow \infty} n^{-1} \Lambda_{n}(n \theta)$ exist, let $I(\alpha)$ be the Fenchel- Legendre transformation of $\Lambda(\theta)$ with $D_{\Lambda}=\{\theta \in \mathbb{R}: \Lambda(\theta)<$ $\infty\}, D_{I}=\{\alpha \in \mathbb{R}: I(\alpha)<\infty\}$, and $D_{\Lambda}^{o}$ and $D_{I}^{o}$ denote their corresponding interiors then, one can state the Gärtner-Ellis theorem, but we need some definitions [7, Section 2.3]. 
Definition 2.4 (Exposed point). A point $\lambda \in \mathbb{R}$ is said to be exposed for $I$ if there is a $y \in \mathbb{R}$ and for all $\alpha \neq \lambda$,

$$
I(\alpha)-I(\lambda)>y \cdot(\alpha-\lambda)
$$

Then $y$ is called an exposing hyperplane of $\lambda$.

Definition 2.5 (Essentially smooth). A convex function $\Lambda$ is said to be essentially smooth if

(i) $D_{\Lambda}$ has nonempty interior $D_{\Lambda^{\prime}}^{o}$

(ii) $\Lambda(\theta)$ is differentiable on $D_{\Lambda^{\prime}}^{o}$

(iii) and $\Lambda(\theta)$ is steep, that is, for any $\theta_{o} \in \partial D_{\Lambda^{\prime}}^{o} \lim _{\theta \rightarrow \theta_{o}}\left|\Lambda^{\prime}(\theta)\right|=\infty$.

The Gärtner-Ellis theorem state that if the limit $\Lambda(\theta)$ exist, then for $\left\{P_{n}\right\}$ the sequence of probability measures on $(\mathbb{R}, \mathcal{B}(\mathbb{R}))$,

(i) for any closed set $F$,

$$
\limsup _{n \rightarrow \infty} n^{-1} \ln P_{n}(F) \leqslant-\inf _{\alpha \in F} I(\alpha),
$$

(ii) for any open set $G$,

$$
\liminf _{n \rightarrow \infty} n^{-1} \ln P_{n}(G) \geqslant-\inf _{\alpha \in G \cap \mathcal{F}} I(\alpha),
$$

where $\mathcal{F}$ is the set of exposed points of $I$ whose exposing hyperplane belongs to $D_{\Lambda}^{o}$.

Suppose in addition that $\Lambda$ is lower semi-continuous function and essentially smooth. Then $\left\{P_{n}\right\}$ obey the LDP with the good rate function $I(\alpha)$.

The Cramér's theorem can be seen as a special case of the Gärtner-Ellis theorem. Because $\bar{X}_{n}$ is the sample mean of the i.i.d. random variables, the limit $\Lambda(\theta)$ in Gärtner-Ellis theorem takes a simple form as the logarithmic of moment generating function of $X_{1}$ given in (2.1) as

$$
\Lambda(\theta)=\ln M(\theta)=\ln E \exp \left\{\theta X_{1}\right\},
$$

then, calculate its Fenchel-Legendre transform as defined in (2.2). To perform calculation one can consider different distributions of $\bar{X}_{n}$, like normal, exponential or Bernoulli distribution see Examples 2.2 and 2.3. 



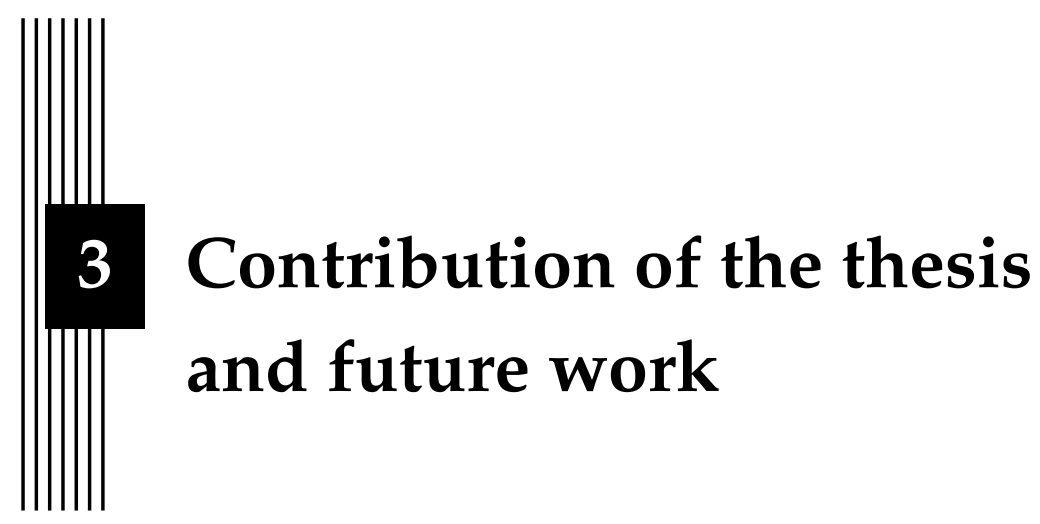

This chapter aims to summarize the contribution of the thesis together with open problems and future work.

\subsection{Contribution of our research}

The contribution of the thesis is to provide the limiting behaviors of condition numbers of suitable $p \times n$ random matrices in term of large deviations with the following main results:

(i) In Paper I, we provide the limiting behaviors of 2-norm condition number of random matrices by taking into consideration the distribution of the i.i.d. entries. To investigate limiting behaviors of condition number in term of large deviations, we relate the large deviation probabilities of condition number to the ones involving $n$ i.i.d. random variables which enables us to consider quite general distribution (namely, sub-Gaussian distribution). Since standard normal distribution is sub-Gaussian, for standard normal entries, we were able to control the upper tail of condition number using the upper tails of ratios of two independent $\chi^{2}$ random variables. We have used the result to establish one application 
in statistical inference: To test the null hypothesis that the covariance is a scalar multiple of identity using union-intersection test method.

The results we provide in Paper I about the asymptotics of $P(k(p, n) \geqslant$ $c)$ with $c \geqslant 1$, as $n \rightarrow \infty$ cannot be found from the existing results in the literature. For instance, the asymptotics cannot be obtained from large deviation asymptotics for extremal eigenvalues given in [13]. The asymptotics with fixed $c$ as $n \rightarrow \infty$ become imprecise from the results established in $[5,12]$ about lower and upper bounds of probability of condition number of $P(k(p, n) \geqslant c)$ with large $c$, fixed $p$ and $n$.

(ii) The spectral properties of Wishart ensembles have been well studied for the cases $\beta=1,2$ or 4 known under the name of real, complex and quaternion Wishart matrices respectively. Because joint distribution of eigenvalues defined in (1.1) is well behaved for every $\beta>0$, the Wishart ensembles have been generalized to $\beta$-Laguerre ensembles or $\beta$-Wishart ensembles for $\beta>0$. A lot of studies about the spectral properties of general $\beta$-Laguerre (or Wishart) ensembles could be found in the literature such as the weak convergence, laws of large numbers and large deviations, which involve all eigenvalues or address first few eigenvalues. Since there is no study on the ratio of extremal eigenvalues in the literature, in Paper II, we provide the study about the limiting behaviors of such ratio in terms of large deviations.

\subsection{Open problems and future work}

To study the asymptotic properties of condition numbers of random matrices, one perspective is to consider the matrix size. The results we provide in Paper I about the study of large deviation probabilities of condition numbers were for some special rectangular random matrices (this is the case when $p<n$ with $p=o(n)$ ). It would be very interesting and challenging to study the limiting behaviors of condition numbers for square random matrices (i.e., $p=n$ ) or for almost square random matrices (i.e., when $n=p+b$ with $b$ being fixed or $b=o(n)$ ) since the several estimates obtained throughout the Paper are not sufficient or precise enough for the cases $p=n$ or $n=p+b$.

Another open problem related to Paper I is to come up with explicit expressions of well defined rate function $I_{\infty, 0}(c)$. In general, it was not easy to find explicit expression of $I_{p, 0}(c)$, but it was possible for some special cases for instance, in Corollary 1.3 the expression of $I_{p, 0}(c)$ independent of $p$ was 
obtained. Therefore, it would be interesting to investigate and to write explicitly the expressions of $I_{\infty, 0}(c)$ as well. If under suitable assumptions one proves that

$$
I_{\infty, 0}(c)=2^{-1} \ln \left[(c+1)^{2} /(4 c)\right],
$$

then this is an universality result that the large deviation asymptotics of the condition number of suitable sub-Gaussian random matrix coincide with the one for Wishart matrices. Here $I_{p, 0}(c)$ and $I_{\infty, 0}(c)$ are well defined rate functions see Section 1.4 Theorem 1.2 and Corollary 1.3.

The last open problem is for both Paper I and II. As mentioned in Section 2.1, for general $p$ and $n$, when $p / n \rightarrow \kappa \in[0,1]$ the condition numbers $k^{2}(p, n)=\lambda_{\max } / \lambda_{\min } \rightarrow\left(1+\kappa^{1 / 2}\right)^{2} /\left(1+\kappa^{1 / 2}\right)^{2}$ in probability. Since the estimates we provide in Paper I and II become inaccurate when $p$ and $n$ are comparable (i.e., $\kappa \in(0,1))$ it would be challenging to investigate the limiting behaviors of $k^{2}(p, n)$ in terms of large deviation when $\kappa \in(0,1)$.

In [19, Theorem 1], about the study of asymptotic properties of the eigenvalues of $\beta$-Laguerre ensembles whose joint probability density is defined as in (1.1), it was shown that the joint probability density of $\beta$-Laguerre ensembles converges to the joint density of $\beta$-Hermite ensembles. In the future research, we would like to study the large deviation asymptotics of condition numbers of general $\beta$-Hermite ensembles. To examine if there are similarities and connections with the results we obtained for $\beta$-Laguerre ensembles. 



\section{Bibliography}

[1] W. Anderson and M. T. Wells. "The exact distribution of the condition number of a Gaussian matrix". In: SIAM Journal on Matrix Analysis and Applications 31.3 (2010), pp. 1125-1130.

[2] Z. D. Bai, J. W. Silverstein, and Y. Q. Yin. "A note on the largest eigenvalue of a large dimensional sample covariance matrix". In: Journal of Multivariate Analysis 26.2 (1988), pp. 166-168.

[3] Z. D. Bai and Y. Q. Yin. "Limit of the smallest eigenvalue of a large dimensional sample covariance matrix". In: Advances In Statistics. World Scientific, 2008, pp. 108-127.

[4] T. H. Baker, P. J. Forrester, and P. A. Pearce. "Random matrix ensembles with an effective extensive external charge". In: Journal of Physics A: Mathematical and General 31.29 (1998), p. 6087.

[5] Z. Chen and J. J. Dongarra. "Condition numbers of Gaussian random matrices". In: SIAM Journal on Matrix Analysis and Applications 27.3 (2005), pp. 603-620.

[6] H. Cramér and H. Touchette (translator). On a new limit theorem in probability theory (Translation of 'Sur un nouveau théorème-limite de la théorie des probabilités'). (accessed May 15, 2020). URL: https : / arxiv . org/ $\mathrm{abs} / 1802.05988$. 
[7] A. Dembo and O. Zeitouni. Large Deviations Techniques and Applications. Springer-Verlag, New York. 1998.

[8] I. Dumitriu. "Eigenvalue statistics for beta-ensembles". PhD thesis. Massachusetts Institute of Technology, 2003.

[9] I. Dumitriu and A. Edelman. "Matrix models for beta ensembles". In: Journal of Mathematical Physics 43.11 (2002), pp. 5830-5847.

[10] F. J. Dyson. "The threefold way. Algebraic structure of symmetry groups and ensembles in quantum mechanics". In: Journal of Mathematical Physics 3.6 (1962), pp. 1199-1215.

[11] A. Edelman. "Eigenvalues and condition numbers of random matrices". In: SIAM journal on matrix analysis and applications 9.4 (1988), pp. 543-560.

[12] A. Edelman and N. R. Rao. "Random matrix theory". In: Acta numerica 14 (2005), pp. 233-297.

[13] A. Fey, R. Van Der Hofstad, and M. J. Klok. "Large deviations for eigenvalues of sample covariance matrices, with applications to mobile communication systems". In: Advances in Applied Probability 40.4 (2008), pp. 1048-1071.

[14] T. Franco. A mini-course in large deviations. (accessed May 27, 2020). URL: http://w3.impa.br/ tertu/archives/LDP_Notes_2015. pdf.

[15] M. I. Freidlin and A. D. Wentzell. "Random perturbations". In: Random perturbations of dynamical systems. Springer, 1998, pp. 15-43.

[16] K. E. Gustafson. Antieigenvalue analysis: with applications to numerical analysis, wavelets, statistics, quantum mechanics, finance and optimization. World Scientific, 2011.

[17] F. den Hollander. Large deviations. Vol. 14. American Mathematical Soc., 2008.

[18] A. T. James. "Distributions of matrix variates and latent roots derived from normal samples". In: The Annals of Mathematical Statistics 35.2 (1964), pp. 475-501.

[19] T. Jiang and D. Li. "Approximation of rectangular beta-Laguerre ensembles and large deviations". In: Journal of Theoretical Probability 28.3 (2015), pp. 804-847. 
[20] K. Johansson. "Shape fluctuations and random matrices". In: Communications in mathematical physics 209.2 (2000), pp. 437-476.

[21] I. M. Johnstone. "On the distribution of the largest eigenvalue in principal components analysis". In: Annals of statistics (2001), pp. 295-327.

[22] A. E. Litvak, A. Pajor, M. Rudelson, and N. Tomczak-Jaegermann. "Smallest singular value of random matrices and geometry of random polytopes". In: Advances in Mathematics 195.2 (2005), pp. 491-523.

[23] R. J. Muirhead. Aspects of multivariate statistical theory. Vol. 197. John Wiley \& Sons, 2009.

[24] D. Petz and F. Hiai. "Logarithmic energy as an entropy functional". In: Contemporary Mathematics 217 (1998), pp. 205-221.

[25] S. Ramasubramanian. "Large deviations: An introduction to 2007 Abel prize". In: Proceedings Mathematical Sciences 118.2 (2008), pp. 161-182.

[26] J. Ramirez, B. Rider, and B. Virág. "Beta ensembles, stochastic Airy spectrum, and a diffusion". In: Journal of the American Mathematical Society 24.4 (2011), pp. 919-944.

[27] M. Rudelson. "Invertibility of random matrices: norm of the inverse". In: Annals of Mathematics (2008), pp. 575-600.

[28] M. Singull, D. Uwamariya, and X. Yang. "Large deviation asymptotics of condition numbers of random matrices". In: Journal of Applied Probability (2021).

[29] M. S. Srivastava and C. G. Khatri. An introduction to multivariate statistics. North-Holland, New York-Oxford, 1979.

[30] T. Tao and V. H. Vu. "Inverse Littlewood-Offord theorems and the condition number of random discrete matrices". In: Annals of Mathematics (2009), pp. 595-632.

[31] L. N. Trefethen and D. Bau III. Numerical linear algebra. Vol. 50. Siam, 1997.

[32] D. Uwamariya and X. Yang. "On the ratio of extremal eigenvalues of $\beta$-Laguerre ensembles". In: Submitted (2021).

[33] S. R. S. Varadhan. "Asymptotic probabilities and differential equations". In: Communications on Pure and Applied Mathematics 19.3 (1966), pp. 261-286.

[34] S. R. S. Varadhan. Large deviations and applications. Vol. 46. SIAM, 1984. 
[35] R. Vershynin. "Introduction to the non-asymptotic analysis of random matrices". In: arXiv preprint arXiv:1011.3027 (2010).

[36] J. Wishart. "The generalised product moment distribution in samples from a normal multivariate population". In: Biometrika (1928), pp. 3252. 


\section{Papers}

The papers associated with this thesis have been removed for copyright reasons. For more details about these see:

http://urn.kb.se/resolve?urn=urn:nbn:se:liu:diva-175751 


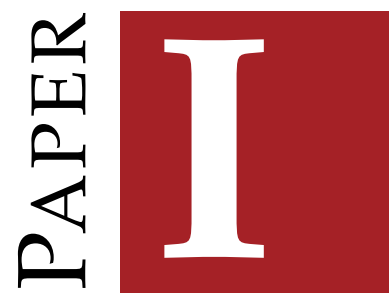

M. Singull, D. Uwamariya, and X. Yang. "Large deviation asymptotics of condition numbers of random matrices". In: Journal of Applied Probability

(2021) 


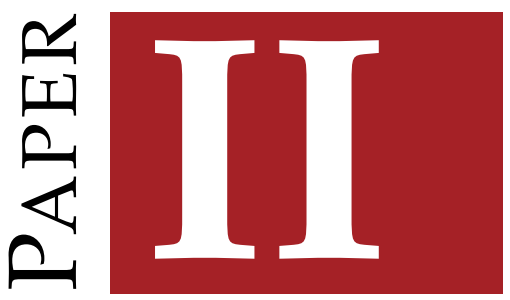

D. Uwamariya and X. Yang. "On the ratio of extremal eigenvalues of $\beta$-Laguerre ensembles". In: Submitted (2021) 


\section{FACULTY OF SCIENCE AND ENGINEERING}

Linköping Studies in Science and Technology, Licentiate thesis No. 1912, 2021 Department of Mathematics

\section{Linköping University}

SE-581 83 Linköping, Sweden

www.liu.se 